\title{
Ischaemic mitral regurgitation
}

\section{Om Prakash Yadava ${ }^{1} \cdot$ Song Wan ${ }^{2}$}

Published online: 2 December 2019

(C) Indian Association of Cardiovascular-Thoracic Surgeons 2019

Looking at semantics as to what qualifies to be called an ischaemic mitral regurgitation (MR), Prof. Wan defines it as one which occurs more than one week after the occurrence of myocardial infarction. Any chronic MR, due to chordal rupture or a leaflet prolapse, should strictly not be labelled as an ischaemic MR. Prof. Wan goes on to discuss the pathophysiology of chronic ischaemic MR and the role of negative left ventricular remodelling in its development. He discusses the concept of leaflet tethering and the pull of the papillary muscles. It is essentially a ventricular problem and the leaflet should not have any structural abnormality. Discussing the decision making in an MR associated with coronary artery disease, Dr. Wan recalls that the guidelines recommend that severe MR should be tackled and, based on the Acker et al. paper in New Engl J Med in 2014, valve should be replaced rather than repaired [1]. However, if a good repair can be carried out, then it may be better than replacement and therefore this decision has to be individualised factoring in the patient, as well as the surgeon.

Discussing decision making in moderate MR, it can be left alone while performing coronary artery bypass grafting (CABG) if it is likely to improve. Therefore, viability testing has a very important role in this subset of patients [2, 3]. However, practically, viability testing, specially magnetic resonance imaging (MRI) based, is difficult to get for most patients because of costs and logistics. Thus if the patient has good distal targets, then the outcomes are likely to be good and viability testing may not be resorted to.

Electronic supplementary material The online version of this article (https://doi.org/10.1007/s12055-019-00877-0) contains supplementary material, which is available to authorized users.

Om Prakash Yadava

op_yadava@yahoo.com

1 National Heart Institute, New Delhi, India

2 Division of Cardiothoracic Surgery, Prince of Wales Hospital, Sha Tin, Hong Kong
The results of surgery for ischaemic MR are quite different from other forms of MR. Two-year recurrence of MR was approaching $60 \%$ in the Acker et al. series [1], which is much higher than the recurrence rate of degenerative MR. Even twoyear mortality was approaching $20 \%$ and is much higher with ischaemic MR, as compared with degenerative MR. Thus, ischaemic MR is a distinct entity, far removed and more complex than degenerative MR. Asked if introduction of percutaneous techniques would change our decision making, Prof. Wan feels that Mitra-clip is an effective solution for functional and ischaemic MR, but lack of a durable annuloplasty system with percutaneous interventions is likely to affect the results, as supporting the annulus is important for any repair technique. But going forward, this will certainly influence our decision making and probably a number of moderate MRs can be left alone during $\mathrm{CABG}$, with a premise that should they progress and become problematic, they can be handled percutaneously.

\section{References}

1. Acker MA, Parides MK, Parrault LP, et al. for the CTSN. Mitral valve repair versus replacement for severe ischaemic mitral regurgitation. New Engl J Med 2014;370:23-32.

2. Penicka M, Linkova H, Lang O, et al. Predictors of improvement of unrepaired moderate ischemic mitral regurgitation in patients undergoing elective isolated coronary artery bypass graft surgery. Circulation. 2009;120:1474-81.

3. Kopjar T, Gasparovic H, Mestres CA, Milicic D, Biocina B. Metaanalysis of concomitant mitral valve repair and coronary artery bypass surgery versus isolated coronary artery bypass surgery in patients with moderate ischaemic mitral regurgitation. Eur J Cardiothorac Surg. 2016;50:212-22.

Publisher's note Springer Nature remains neutral with regard to jurisdictional claims in published maps and institutional affiliations. 